\title{
Slanted hole array beam profiler (SHArP)—a high-resolution portable beam profiler based on a linear aperture array
}

\author{
Xiquan Cui, Xin Heng, Jigang Wu, Zahid Yaqoob, Axel Scherer, Demetri Psaltis, and Changhuei Yang \\ Department of Electrical Engineering, California Institute of Technology, Pasadena, California 91125 \\ Received May 17, 2006; revised August 11, 2006; accepted August 16, 2006; \\ posted August 22, 2006 (Doc. ID 71106); published October 11, 2006

\begin{abstract}
We demonstrate a novel high-resolution portable beam profiler based on a slanted linear array of small apertures, termed a slanted hole array beam profiler (SHArP). The apertures are directly fabricated on a metal-coated CMOS imaging sensor. With a single linear scan, the aperture array can establish a virtual grid of sampling points for beam profiling. With our prototype, we demonstrate beam profiling of Gaussian beams over an area of $66.5 \mu \mathrm{m} \times 66.5 \mu \mathrm{m}$ with a resolution of $0.8 \mu \mathrm{m}$ (compare with the CMOS pixel size of $10 \mu \mathrm{m})$. The resolution can be improved into the range of submicrometers by fabricating smaller apertures. The good correspondence between the measured and calculated beam profiles proves the fidelity of our new beam profiling scheme. (C) 2006 Optical Society of America
\end{abstract} \\ OCIS codes: $110.0180,230.3990$.
}

The quantitative measurement of laser beam profiles is important for ensuring the efficient and accurate use of lasers in applications ranging from laser machining to fiber optics to LASIK surgery. ${ }^{1}$ In addition, precise knowledge of the focal field distribution of high-NA lenses is important in the design of systems such as confocal laser scanning microscopy and optical serial sectioning microscopy. ${ }^{2}$ Commercial beam profilers based on 2D imaging sensor arrays, e.g., CCDs or CMOSs, have difficulties addressing this need, as their resolution is limited by the pixel size of the underlying sensor array (typically $\sim 5 \mu \mathrm{m}$ or more)..$^{3}$

There are two major types of high-resolution beam profiler. The first type is based on a scanning knife edge or slit and is well suited for high-resolution profiling of symmetric beams. ${ }^{4}$ The second type is based on the use of sharp probe tips ${ }^{5}$; its raster-scan nature is suited for arbitrary beam profiles, but the scans can be time consuming.

In this Letter we present a novel high-resolution portable beam profiler - the slanted hole array beam profiler (SHArP) - that consists of a slanted linear array of small apertures on a metal-coated CMOS imaging sensor. To improve the resolution of a conventional CCD or CMOS beam profiler, smaller sensor pixels will need to be fabricated. However, fabrication limitations as well as cross talk between pixels impose a practical limit on the minimum sensor pixel size. In the SHArP these two issues are circumvented by using small apertures etched through the metal layer to limit the light sensitive area of the underlying pixel. The SHArP's resolution is defined by the aperture size.

The novelty of the SHArP method can be illustrated as follows. Consider a 2D metal coated sensor grid in which a small aperture is etched through the metal layer at the center of each pixel. While we can achieve a smaller effective pixel size this way, the images obtained will be sparsely pixelated. One approach to obtain high resolution and filled-out images will be to raster scan the sensor grid; the timevarying signal collected through all the apertures can be used to form a high-resolution beam image. The concept in SHArP follows the same general idea. However, we employ a linear array of apertures instead of a 2D array. Further, we replace the raster scan with a simple linear one. With a sufficiently long array and appropriate aperture size, we can profile a beam of any size with high resolution. This method can also be viewed as a parallelized implementation of the sharp probe tip method with two key differences-the replacement of raster scan with a much simplified linear scan and the potential for parallel beam profiling. Finally, we note that this imaging method is conceptually similar to the optofluidic microscope. ${ }^{6}$

Figure 1(a) shows the operation of the SHArP. First a linear array of apertures is fabricated on a metal-coated linear CMOS sensor grid; each aperture maps uniquely onto an individual pixel. Then the SHArP is linearly translated across the target light beam with a uniform speed $(v)$. The linear aperture array is oriented at a small angle $(\theta)$ with respect to the translation axis $X$. During the translation, each aperture will sample a line profile of the beam in the scanning direction $X$. The angle tilt ensures that each aperture scans a different line on the light beam. The composition of the line scans constitutes an effective sensor grid for sampling the whole target light beam.

We note that, as the beam passes the apertures sequentially, there is a constant time delay between adjacent line profile recordings, which is equal to the time interval in which the beam travels across two adjacent apertures [see Fig. 1(b)]. The time delay $t_{\text {delay }}$ is given by

$$
t_{\text {delay }}=\frac{\Delta X}{v}=\frac{L \cos \theta}{v},
$$

where $L$ is the distance between adjacent apertures, $\Delta X$ is the projection of $L$ on the scanning direction $X$, 


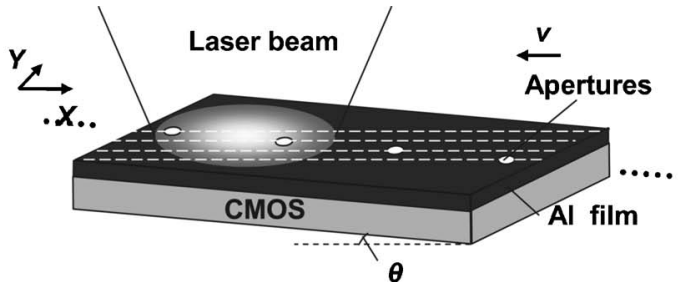

(a)

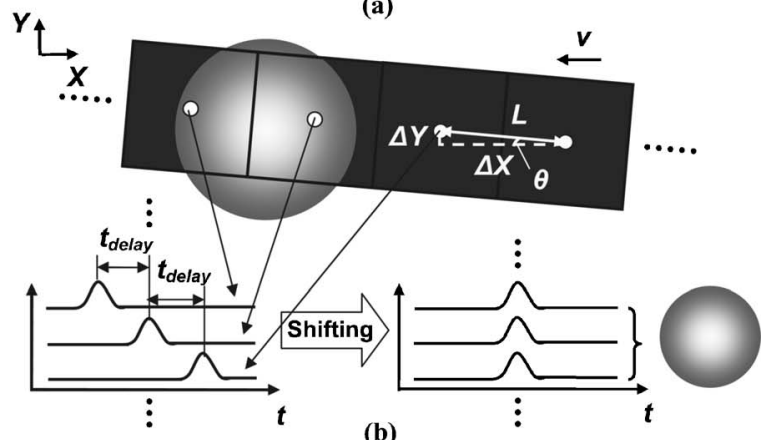

(b)

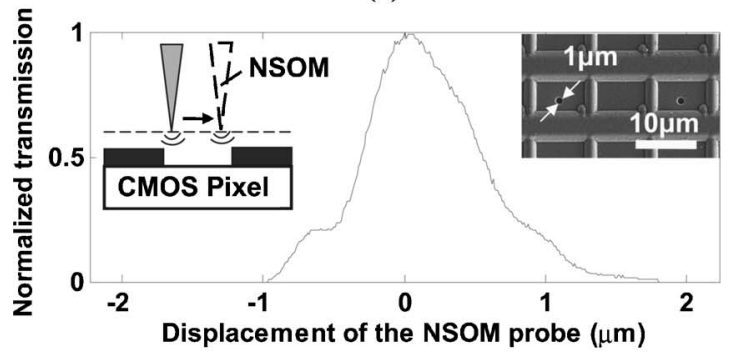

(c)

Fig. 1. (a) Principle of the SHArP. (b) Top view and image reconstruction of the SHArP. (c) PSF of the SHArP prototype measured by a near-field scanning optical microscope.

and $v$ is the translation speed of the entire SHArP device. By shifting the acquired line profiles with this delay, we can then obtain an accurate profile of the whole beam.

The effective pixel grid spacing of the measured beam profile by the SHArP is determined by the distance between the adjacent sampling points. In the $Y$ direction, this is equal to the spacing between adjacent apertures along the $Y$ direction $\delta Y=\Delta Y=L \sin \theta$. In the $X$ direction, it is equal to the distance traveled by the SHArP between two subsequent sampling instances in the $X$ direction, $\delta X=v / f$, where $f$ is the frame rate of the CMOS sensor.

We fabricated the prototype SHArP on a 640 $\times 480$ CMOS imaging sensor (Micron Technology, MT9V403). The pixel size is $9.9 \mu \mathrm{m} \times 9.9 \mu \mathrm{m}$. A $200 \mathrm{~nm}$ thick aluminum film, which serves as an optically opaque mask, was coated onto the surface of the CMOS sensor by thermal evaporation. Then a dual-beam focused ion beam machine (Nova 200, FEI Company) was used to mill 128 holes through the film at the centers of pixels in a row [see the inset in Fig. 1(c)]. Each aperture had a diameter of $1.0 \mu \mathrm{m}$. The adjacent apertures were created on every other CMOS pixel. We previously established that the transmission through each aperture leaked through to adjacent pixels (the leakage is measured at $-10 \mathrm{~dB}$ with respect to the transmission to the main receiving pixel). Thus we spaced the apertures out to miss every other pixel to reduce cross talk between adja- cent apertures. Finally, we note that, while the employed CMOS sensor array was $2 \mathrm{D}$, only output signals from the relevant line of pixels were acquired. The line rate of the CMOS sensor was $\sim 0.9 \mathrm{kHz}$. The SHArP method is optimally implemented on a linear sensor array.

To determine the resolution limit of our SHArP prototype, we used a near-field scanning optical microscope (Alpha-SNOM, WITec GmbH). We scanned the microscope probe across one of the apertures on the SHArP with a probe-sample distance less than $10 \mathrm{~nm}$ and recorded the light transmission with the underlying CMOS pixel [see Fig. 1(c)]. The normalized transmission curve represents the point spread function (PSF) of the SHArP (FWHM $0.9 \mu \mathrm{m}$ ). The measured resolution limit, which we define as the minimum displacement between two overlapping PSFs such that the two peaks are still resolvable, is $0.8 \mu \mathrm{m}$. We note that the variation in the PSF profile in Fig. 1(c) is attributable to imperfections in the fabricated aperture. Finer fabrication control should improve this.

We also note that the transmissions through the holes do vary from hole to hole because of fabrication variations. To normalize the transmissions through the holes with respect to one another, we uniformly illuminated the entire hole array and calibrated them by measuring the resulting transmissions.

We draw attention to the difference between pixel grid spacing and effective SHArP pixel size. The pixel grid spacing is given by $\delta X$ and $\delta Y$; they can be adjusted by changing the tilt angle $(\theta)$ and SHArP scan speed $(v)$ during the experiment. They are also related to the underlying CMOS pixel separation $(L)$ and CMOS frame rate $(f)$ - variables that are not changeable in the experiment. The effective SHArP pixel size is given by the measured resolution limit $(0.8 \mu \mathrm{m}$ in the prototype). When $\delta X$ and $\delta Y$ are chosen to be equal to the SHArP's pixel size, the SHArP will be equivalent to an ideal $100 \%$ fill factor CCD or CMOS 2D sensor. For our prototype, this condition requires $\theta=0.050 \mathrm{rad}$ and $v=920 \mu \mathrm{m} / \mathrm{s}$. However, it is generally desirable to have an overfilled virtual grid (grid spacing is smaller than the pixel size), as it leads to a higher-density sampling of the target beam profile and suppreses aliasing artifacts. ${ }^{7}$

To evaluate our prototype, we performed beam profiling experiments. We coupled light from a $635 \mathrm{~nm}$ laser source (Thorlabs S1FC635) into Corning Puremode HI 780-5/125 fiber. Output light from the optical fiber was collimated by a lens with a $11 \mathrm{~mm}$ focal length (Thorlabs C220TM-B). The measured collimated beam waist diameter of the collimated beam was $1.66 \mathrm{~mm}$ (The $1 / e^{2}$ beam waist diameter is used throughout this Letter). Then the collimated beam was focused by a lens similar to that used for collimation. The SHArP was located at the focal plane of the second lens. The power incident on the beam profiler was $10 \mu \mathrm{W}$. We scanned the SHArP across the focused beam spot in the $X$ direction with an $X Y$ translation stage driven by a Newport CMA-25CCCL linear actuator. The scanning speed of the beam profiler was $103 \mu \mathrm{m} / \mathrm{s}$, and $\theta$ was $0.011 \mathrm{rad}$; the resulting 
pixel grid spacing dimensions $\delta X$ and $\delta Y$ were 0.11 and $0.22 \mu \mathrm{m}$, respectively. Figure 2(a) shows the $28.4 \mu \mathrm{m} \times 28.4 \mu \mathrm{m}$ beam profile with 255 $\times 128$ pixels recorded by the SHArP. The acquisition took $\sim 25 \mathrm{~s}$. The measured and calculated beam waist diameters were 5.4 and $5.3 \mu \mathrm{m}$, respectively. For comparison, the cross sections of the measured beam profile (circles) and the calculated ideal Gaussian beam profile (in solid curve) in $X$ and $Y$ directions are plotted in Figs. 2(e) and 2(f). As a comparison with a conventional 2D imaging-sensor-based beam profiler, we projected the beam directly onto some uncoated pixels of the same CMOS sensor. Owing to the narrow waist of the beam, only one CMOS pixel detected a signal, Fig. 2(b). This demonstrates that the SHArP can achieve a higher resolution than a conventional 2D imaging-sensor-based beam profiler.

Next, we placed an iris diaphragm (Thorlabs SM1D12) between the two lenses to limit the diameter of the collimated beam. Figure 2(c) shows the fo-
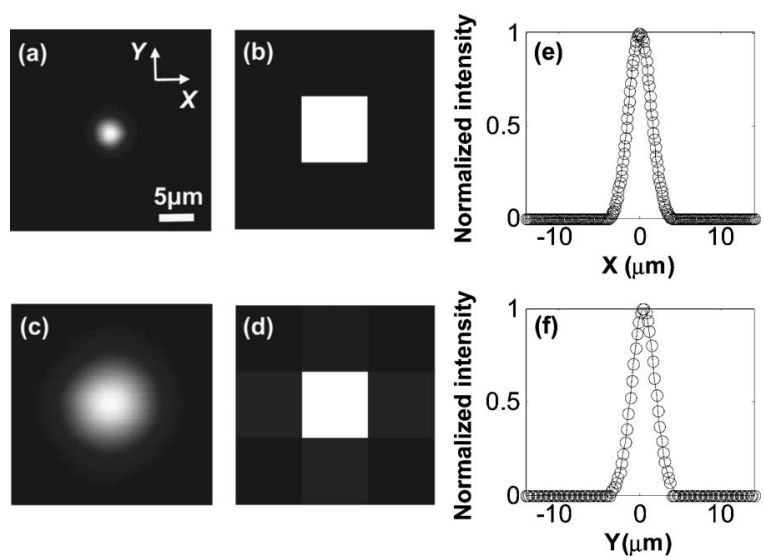

Fig. 2. Focal beam profile of the Gaussian beam (a) measured by the SHArP, (b) imaged by the CMOS imaging sensor. Focal beam profile of the Gaussian beam confined by an iris diaphragm in width (c) measured by SHArP, (d) imaged by the CMOS imaging sensor. The cross section of the focal beam profile of the Gaussian beam (e) in the $X$ direction and (f) in the $Y$ direction (measured, circles; calculated, solid curve).

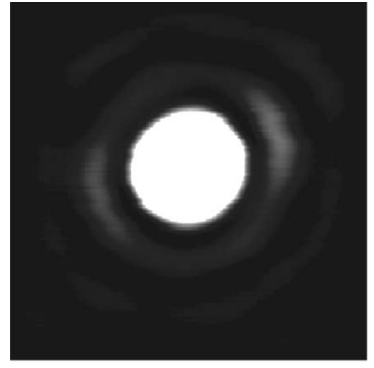

(a)

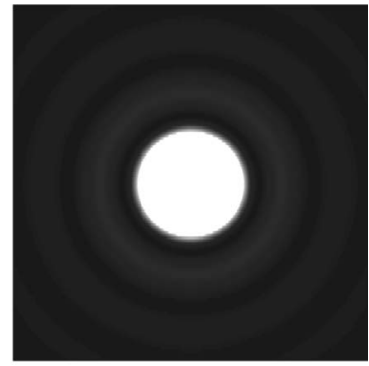

(b)
Fig. 3. Focal diffraction pattern of the Gaussian beam confined by an iris diaphragm in width (a) measured by the SHArP, (b) calculated (zeroth order has been truncated). cused beam profile recorded by the SHArP when the diameter of the collimated beam was limited by the iris diaphragm to about $0.8 \mathrm{~mm}$. As the SHArP was at the focal plane of the second lens, the focused beam showed a Fraunhofer diffraction pattern of the confined beam. The measured and calculated beam waist diameters were 14.2 and $14.6 \mu \mathrm{m}$, respectively. Figure 2(d) shows the beam profile of the same focused beam imaged directly by the uncoated pixels on the same CMOS sensor. Only 5 pixels yielded a readout signal.

To see the weak high-order terms of the diffraction pattern around the zeroth order of the confined beam profile, we increased the power of the beam to $140 \mu \mathrm{W}$. Because of the finite number of apertures fabricated in our SHArP prototype, the larger tilt angle $\theta=0.026 \mathrm{rad}$, was used to enlarge the range of beam profiling to $66.5 \mu \mathrm{m} \times 66.5 \mu \mathrm{m}$. The scanning speed of the beam profiler was $103 \mu \mathrm{m} / \mathrm{s}$. The resulting pixel grid spacing dimensions $\delta X$ and $\delta Y$ were 0.11 and $0.52 \mu \mathrm{m}$, respectively. Figure 3(a) shows the SHArP measured beam profile $(583 \times 128)$. We note that the first and second orders of the diffraction pattern surrounding the saturated zeroth order are clearly visible and that they match the theoretical predictions, Fig. 3(b). The average measured and calculated first diffraction order radii were 17.0 and $17.2 \mu \mathrm{m}$, respectively. The asymmetric first- and second-order diffraction rings are attributable to the asymmetry of the iris diaphragm.

In summary, we have presented a new type of beam profiler, based on a linear aperture array, that allows profiling of arbitrary beam shapes with high resolution. A $25 \mathrm{~s}$ beam profiling over a $66.5 \mu \mathrm{m}$ $\times 66.5 \mu \mathrm{m}$ range with a $0.8 \mu \mathrm{m}$ resolution limit has been implemented. The resolution can be improved by using even smaller, submicrometer, apertures.

We acknowledge financial support from the Defense Advance Research Projects Agency Center for Optofluidic Integration. Xiquan Cui's e-mail address is xiquan@caltech.edu.

\section{References}

1. A. Guirao, D. R. Williams, and S. M. MacRae, J. Refract. Surg. 19, 15 (2003).

2. S. F. Gibson and F. Lanni, J. Opt. Soc. Am. A 8, 1601 (1991).

3. S. K. Rhodes, A. Barty, A. Roberts, and K. A. Nugent, Opt. Commun. 145, 9 (1998).

4. W. Plass, R. Maestle, K. Wittig, A. Voss, and A. Giesen, Opt. Commun. 134, 21 (1997).

5. S. K. Rhodes, K. A. Nugent, and A. Roberts, J. Opt. Soc. Am. A 19, 1689 (2002).

6. X. Heng, D. Erickson, L. R. Baugh, Z. Yaqoob, P. W. Sternberg, D. Psaltis, and C. Yang, Lab Chip 10.1039/ b604676b (2006).

7. S. K. Park and R. A. Schowengerdt, Appl. Opt. 21, 3142 (1982). 